\title{
Phospholipid compositional changes of five pseudomonad archetypes grown with and without toluene
}

Received: 13 October 1999 / Received revision: 16 February 2000 / Accepted: 25 February 2000

\begin{abstract}
Bacterial physiological responses to toluene exposure were investigated in five reference pseudomonad strains that express different toluene degradation pathways: Pseudomonas putida $\mathrm{mt}-2$, Pseudomonas putida F1, Burkholderia cepacia G4, Burkholderia pickettii PKO1, and Pseudomonas mendocina KR1. The intact phospholipids of these archetypes, grown with and without toluene, were characterized using liquid chromatography/electrospray ionization/mass spectrometry. All strains showed significant changes in phospholipid content and composition as an adaptive response to toluene exposure, as well as considerable diversity in response mechanisms. For example, the phospholipid content of toluene-grown PKO1, F1, and KR1 were $10.9-34.7 \%$ of that found in succinate-grown strains, while the phospholipid content of $\mathrm{mt}-2$ and G4 increased by $56 \%$ and $94 \%$, respectively, when grown on toluene. In addition, PKO1, F1, and mt-2 responded to the presence of toluene by synthesizing more phosphatidylglycerol, whereas G4 and KR1 synthesized phospholipids with polyunsaturated fatty acids $(\mathrm{C} 18: 2)$ on one or both of the $s n-2$ positions. These changes in phospholipid composition and concentration probably reflect the sensitivity and degree of tolerance of these strains to toluene, and suggest that different mechanisms are utilized by dissimilar bacteria to maintain optimal lipid ordering in the presence of such environmental pollutants.
\end{abstract}

\footnotetext{
J. Fang $(\bowtie) \cdot$ M. J. Barcelona

Department of Civil and Environmental Engineering,

The University of Michigan, Ann Arbor, MI 48109-2099, USA

e-mail: jsfang@engin.umich.edu

Tel.: + 1-734-9363177

Fax: + 1-734-7636513

P. J. J. Alvarez

Department of Civil and Environmental Engineering,

The University of Iowa, Iowa City, IA 52242-1527, USA
}

\section{Introduction}

The monoaromatic hydrocarbons, benzene, toluene, ethylbenzene, and xylenes (BTEX) are common environmental contaminants that represent a serious threat to ground water resources and to public health (Tursman and Cork 1992). "Bioremediation" is the overall term applied to the mitigation of environmental pollution by microbial populations capable of conducting biodegradation processes. Engineered bioremediation and monitored natural attenuation hold great promise as an approach to clean up BTEX-contaminated aquifers (National Research Council 1993). Nevertheless, process optimization is limited by our incomplete understanding of microbial population, metabolic, and physiologic shifts due to the presence of BTEX compounds. Understanding the diversity of such adaptation mechanisms is important to delineate the capabilities and limitations of different bioremediation strategies.

Microbial activities depend on adaptive responses to priority pollutants, including changes in membrane lipid composition. Several researchers have studied such adaptation mechanisms to alcohols and aromatic hydrocarbons (Bansal and Khuller 1981; Barrow et al. 1983; Guckert et al. 1986; Heipieper and de Bont 1994; Heipieper et al. 1992; Ingram 1976, 1977, 1986; Weber et al. 1994, 1996; Pinkart and White 1997). Demonstrated mechanisms of physiologic adaptation include increased synthesis of saturated (Ingram 1986) or unsaturated fatty acids (Beavan et al. 1982; Dombek and Ingram 1984), conversion of cis- to trans-unsaturated fatty acids (Heipieper and de Bont 1994; Heipieper et al. 1992; Weber et al. 1994), and possibly alterations of phospholipid head groups (Melchior 1982). However, comparative studies that address the diversity of such adaptive responses among different strains capable of degrading a given toxic chemical are lacking.

In this study, we used intact phospholipid profiling by liquid chromatography/electron spray ionization/ mass spectrometry (LC/ESI/MS) to characterize cell 
membrane phospholipid profiles for five reference pseudomonad strains that express the five known aerobic toluene degradation pathways (Fig. 1). In doing so, information was obtained about the diversity of structural changes in the cell membrane as a response to toluene exposure.

\section{Materials and methods}

Bacterial cultures

Pseudomonas putida mt-2, Pseudomonas putida F1, Burkholderia cepacia G4, Burkholderia pickettii PKO1, and Pseudomonas mendocina KR1 were selected for the application of microbial phospholipid profiling. These reference strains represent the archetypes of the five aerobic degradation pathways of toluene (Fig. 1). All cells were grown at about $25^{\circ} \mathrm{C}$ on mineral salts base medium (Stanier et al. 1966) amended with either $10 \mathrm{mM}$ succinate or toluene. Toluene was introduced in the vapor phase by a bulb suspended from the flask stopper, as described by Gibson et al. (1973).

\section{Lipid extraction}

Total lipids were extracted with a modified Bligh and Dyer extraction method (Fang and Findlay 1996; White et al. 1979). Approximately $6 \mathrm{ml}$ liquid bacteria culture was added to a test tube filled with $22.5 \mathrm{ml}$ methanol, dichloromethane (DCM), and phos- phate buffer (2:1:0.8) extraction solution. The extraction mixture was allowed to stand overnight in darkness at $4{ }^{\circ} \mathrm{C}$. The lipids were then partitioned by adding DCM and water such that the final ratio of DCM to methanol to water was 1:1:0.9. The upper aqueous phase was discarded and the lower organic phase was then decanted through a cellulose \#4 filter into a test tube. The solid residue retained on the filter was washed with $3 \times 1 \mathrm{ml}$ DCM. The total lipid extract was dried under a gentle stream of nitrogen and was once again dissolved in methanol. Total lipids were separated into different lipid classes using miniature champagne columns (Supelco Inc., Bellefonte, Pa.). Neutral lipids, glycolipids, and phospholipids were obtained by eluting with $4 \mathrm{ml}$ chloroform, acetone, and methanol, respectively (Fang and Findlay 1996).

Liquid chromatography/electrospray ionization/mass spectrometry

The LC/ESI/MS analysis was performed on an HP 1090 liquid chromatograph/HP 5989B single quadrupole mass spectrometer with an electrospray interface. The LC was equipped with a $250-\mu 1$ sample loop. A HP reverse phase HPLC column (Zorbax $150 \mathrm{~mm} \times 4.6 \mathrm{~mm}, 5 \mu \mathrm{m}$ ) was used for the chromatographic separation of phospholipids. A gradient solvent system composed of solvent A (10 mM ammonium acetate), solvent B (methanol), and solvent $\mathrm{C}$ (acetonitrile) was used with a flow rate of $0.5 \mathrm{ml} / \mathrm{min}$. At the beginning of the gradient, the mobile phase was $25 \%$ of $\mathrm{A}$ and $75 \%$ of B for 2 min. Solvent B was increased to $90 \%$ and solvent C increased to $10 \%$ at $30 \mathrm{~min}$. The mobile phase was then held isocratically for $5 \mathrm{~min}$.

The mass spectrometer was operated in the negative ionization mode. Nitrogen drying gas flow was approximately $12 \mathrm{l} / \mathrm{min}$ and its temperature was set at $300{ }^{\circ} \mathrm{C}$. The electrospray needle was held at
Fig. 1 Aerobic toluene degradation pathways encoded by five pseudomonad strains. Enzyme designations: 1A, xylene oxygenase (or toluene oxidase); 2A, benzyl alcohol dehydrogenase; $3 \mathrm{~A}$, benzaldehyde dehydrogenase; 4A, toluate 1,2-dioxygenase; $5 \mathrm{~A}$, dihydroxycyclohexadiene carboxylate dehydrogenase; 6A, catechol 2,3-oxygenase; 1B, toluene dioxygenase; $2 \mathrm{~B}$, toluene dihydrodiol dehydrogenase; 3B, 3-methylcatechol 2,3-dioxygenase; $1 \mathrm{C}$, toluene 2 (ortho)-monooxygenase; 2C, 3-methylcatechol-2,3-dioxygenase; 1D, toluene 3 (meta)monooxygenase; $2 \mathrm{D}$, phenol hydroxylase; 3D, catechol 2,3oxygenase; $1 \mathrm{E}$, toluene 4 (para)monooxygenase; $2 \mathrm{E}$, $p$-cresol methylhydroxylase; 3E, $p$-hydroxybenzaldehyde dehydrogenase; $4 \mathrm{E}, p$-hydroxybenzoate hydroxylase; $5 \mathrm{E}$, protocatechuate-3,4-dioxygenase

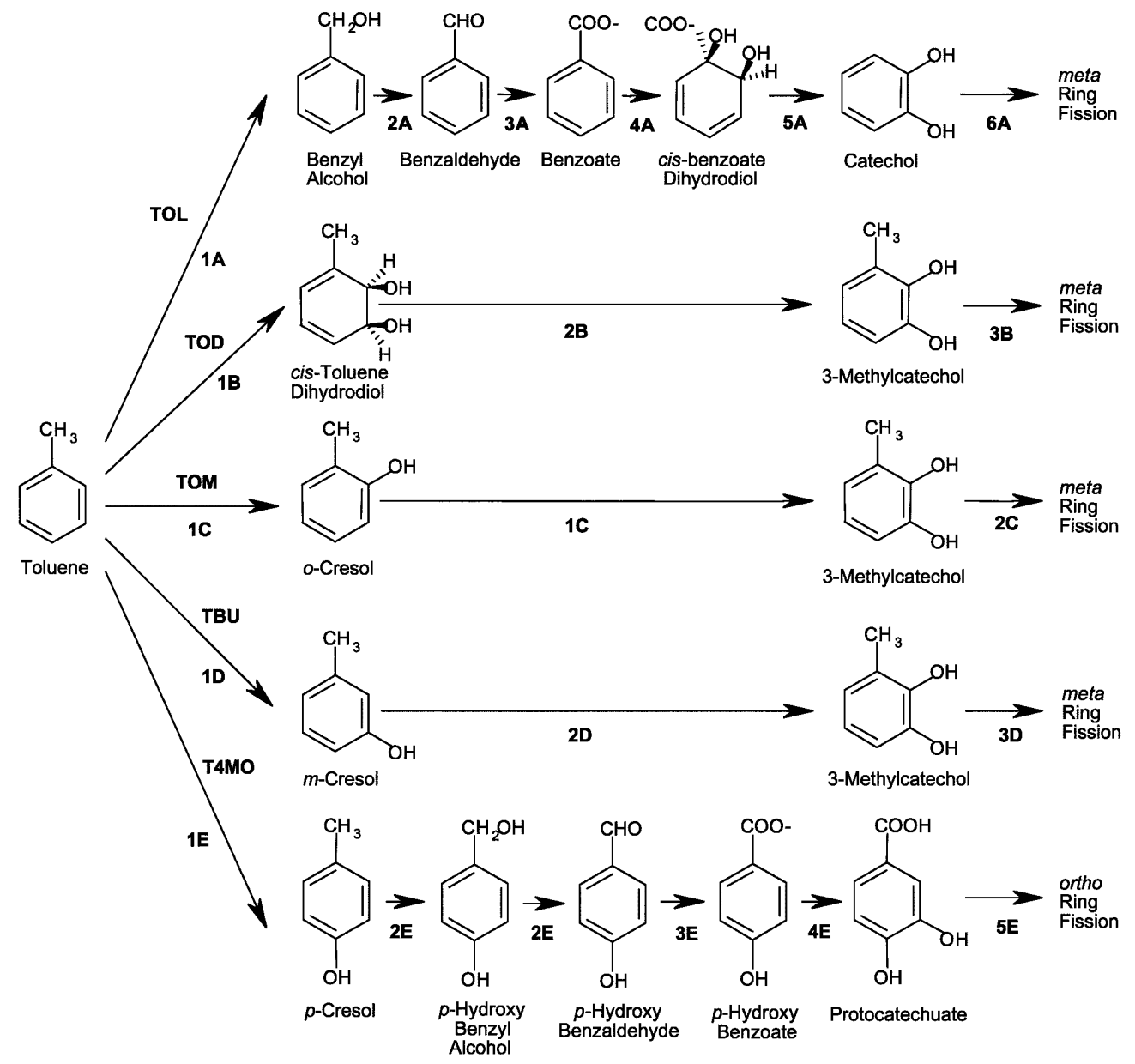


ground potential, and the capillary, end plate, and cylinder voltages were set at 3500,2500 , and $1500 \mathrm{~V}$, respectively, and maintained at high positive potential for negative ionization. An Iris hexapole ion guide (Analytica of Bradford, Bradford, Conn.) in the MS source enhanced the efficiency of ion transfer and the sensitivity of the mass spectrometer. The capillary exit voltage was set at $200 \mathrm{~V}$ at all times. The mass spectrometer was tuned using a solution provided by the manufacturer. The mass spectrometer was scanned from 70 to $1000 \mathrm{amu}$ at approximately $0.4 \mathrm{scan} / \mathrm{s}$. The concentrations of phospholipids were calculated based on chromatographic area response of individual phospholipid relative to that of internal standard (18:1-lyso-phosphatidylglycerol) and reported as $\mu \mathrm{g} / \mathrm{ml}$ liquid culture. The reproducibility of the analysis was better than $87 \%(n=5)$.

Phospholipids were designated as follows: $\mathrm{C} 1 \mathrm{~d} 1 / \mathrm{C} 2 \mathrm{~d} 2-\mathrm{PL}$ (e.g., $\mathrm{C} 16: 0 / \mathrm{C} 18: 1-\mathrm{PS}$ ), where $\mathrm{C} 1$ and $\mathrm{C} 2$ are the number of carbon atoms in the fatty acyl chains on the $s n-1$ and $s n-2$ positions, respectively; $\mathrm{d} 1$ and $\mathrm{d} 2$ are the number of double bonds of the $s n-1$ and $s n-2$ fatty acyl chains, respectively; and PL is the abbreviation for phospholipids (see Table 1).

\section{Phospholipid response index}

A new index, called the phospholipid response index (PRI), was formulated to characterize the bacterial responses in membrane lipids to toluene exposure, including possible changes in classes of phospholipids (head group composition), fatty acyl chain length, and degree of unsaturation. The PRI is arbitrarily defined as:

$\mathrm{PRI}=\left(\mathrm{N}_{\mathrm{CL} 1} \times \mathrm{DS}_{1}+\mathrm{N}_{\mathrm{CL} 2} \times \mathrm{DS}_{2}\right) / 2+\mathrm{N}_{\mathrm{HG}}$

where $\mathrm{N}_{\mathrm{CL} 1}$ and $\mathrm{N}_{\mathrm{CL} 2}$ are the chain-length numbers of the fatty acids on $s n-1 / s n-2$ positions, respectively. $\mathrm{N}_{\mathrm{CL}}$ is equal to 20 minus the length of the corresponding fatty acid chain. For example, for a C16 fatty acid, the chain length number equals $20-16=4$. Thus, higher $\mathrm{N}_{\mathrm{CL}}$ values correspond to shorter fatty acid chains that contribute to loose packing of acyl chains in the phospholipid bilayer and increase membrane fluidity (Pluschke and Overath 1981).

$\mathrm{DS}_{1} / \mathrm{DS}_{2}$ is the degree of saturation of the fatty acids on $s n-1 /$ $s n-2$ positions, respectively. $\mathrm{DS}=1$ for saturated fatty acids, $\mathrm{DS}=5$ for monounsaturated fatty acids, and DS $=10$ for polyunsaturated fatty acids. Higher DS values reflect the fact that unsaturation increases the molecular cross-sectional area, which also contributes to loose packing of acyl chains and higher membrane fluidity (Stubbs et al. 1981).

$\mathrm{N}_{\mathrm{HG}}$ is the headgroup number, with values assigned as follows: $\mathrm{N}_{\mathrm{HG}}=10$ for phosphatidylglycerol (PG), and $\mathrm{N}_{\mathrm{HG}}=2$ for phosphatidylethanolamine (PE) or phosphatidyldimethylethanolamine (PDME). A higher $\mathrm{N}_{\mathrm{HG}}$ value was selected for $\mathrm{PG}$ based on the fact that, given identical fatty acid compositions, PG has a bigger sectional area than PE and PDME in lipid bilayers and therefore its presence contributes to loose packing of acyl chains in lipid bilayers and greater membrane fluidity (Cronan and Rock 1987; Weber et al. 1996).

Thus, PRI is a comprehensive index accounting for the head group composition, the degree of unsaturation, and the length of fatty acids in a phospholipid. The larger the PRI, the less ordering of the membrane phospholipids (short chain length, unsaturated fatty acids, bigger head groups, etc.) and the more fluid the cell membrane. The PRI of each strain was calculated according to Eq. 1, and represents the average of the PRI of each phospholipid molecule detected in the strain.

\section{Results}

The phospholipid profiles and composition of the bacterial strains grown with and without toluene are shown in Fig. 2 and Table 1. Phospholipids were identified based on their mass spectra in the negative ionization
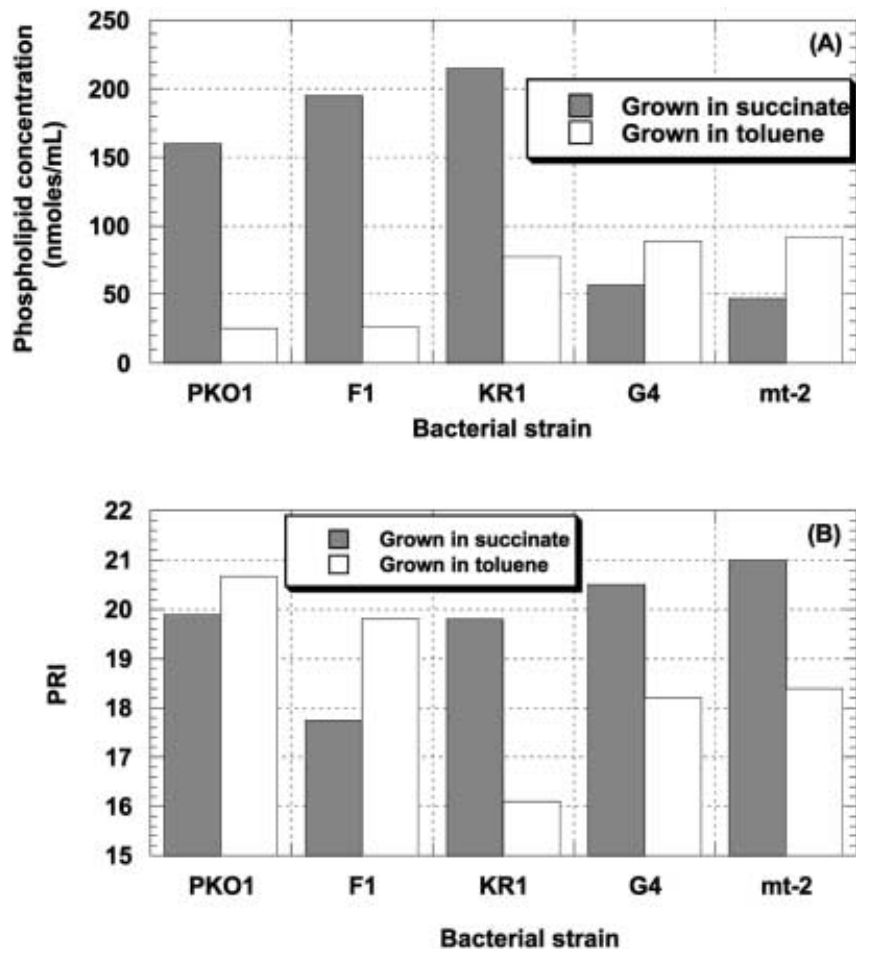

Fig. 2A, B Total ion chromatogram of phospholipids from five pseudomonad strains grown in the absence $(\mathbf{A})$ or presence $(\mathbf{B})$ of toluene: PKO1, B. pickettii $\mathrm{PKO} 1 ; \mathrm{mt}-2$, P. putida $\mathrm{mt}-2$; F1, P. putida F1; G4, B. cepacia G4; KR1, P. mendocina KR1. For compound identification (peak numbers), see Table 1

mode (Fang and Barcelona 1998). Positions of fatty acids on the $s n$ positions were determined based on the ratio of intensity of fragment ions representing each fatty acid (Fang and Barcelona 1998). Fatty acids with the same degree of unsaturation but different positions of double bonds were not differentiated. Twenty-two phospholipids in three different classes were identified in the five reference strains (Table 1). Fatty acids on the $s n-1$ and $s n-2$ positions of the phospholipids were either saturated, monounsaturated, or diunsaturated with chain length from 14 to 18 . The phospholipid content from each strain varied appreciably, as did the composition of the strains (Table 1).

\section{Phospholipid profiles of Pseudomonas putida mt-2}

Major phospholipids identified in succinate-grown mt-2 were PE, PDME, and PG (Table 1; Fig. 1). All except two phospholipids were asymmetrical - with two different fatty acids on the $s n-1$ and $s n-2$ positions (Table 1). Interestingly, fatty acids on the symmetrical phospholipids were all monounsaturates, either $\mathrm{C} 16: 1$ or $\mathrm{C} 18: 1$. No phospholipids with odd-numbered fatty acids were detected in succinate-grown bacteria. The major phospholipids were 16:0/16:1-PG (35.6\%) and 18:1/16:1-PE $(29.0 \%)$, whereas the symmetrical phospholipids $16: 1 /$ 16:1-PE and 16:1/16:1-PDME combined constituted $20.9 \%$ of the total phospholipid content (Table 1). 


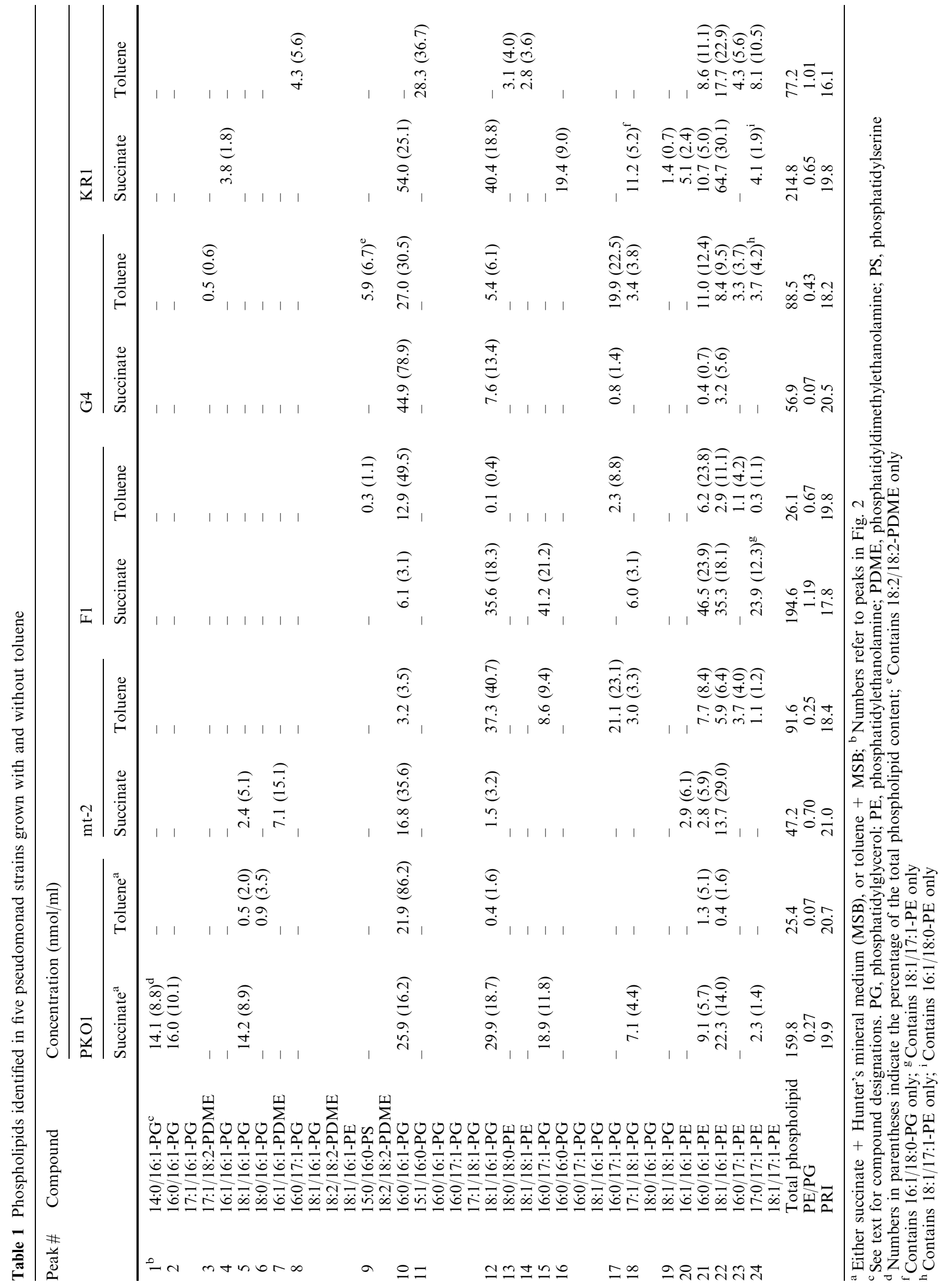


Growth of mt-2 on toluene resulted in significant changes in the relative abundance of different phospholipids (Table 1; Fig. 2). Some phospholipids (e.g., 18:1/16:1-PG, 16:1/16:1-PE, and 16:1/16:1-PDME) disappeared, whereas new ones were synthesized $(16: 0 /$ 17:1-PG, 17:1/18:1-PG, 16:0/17:1-PE, 17:0/17:1-PE, and
18:1/17:1-PE). All of the newly synthesized phospholipids contained the $\mathrm{C} 17$ fatty acids. The concentration ratio of total PE over total PG decreased from 0.70 to 0.25 , whereas the total concentration of phospholipids increased by $94.1 \%$ (from 47.2 to $91.6 \mathrm{nmol} / \mathrm{ml}$; Fig. 3A). The PRI decreased from 21.0 to 18.4
Fig. 3A, B Changes in total phospholipid content (A) and phospholipid response index (PRI) (B) in five pseudomonad strains grown in the presence or absence of toluene
(A)
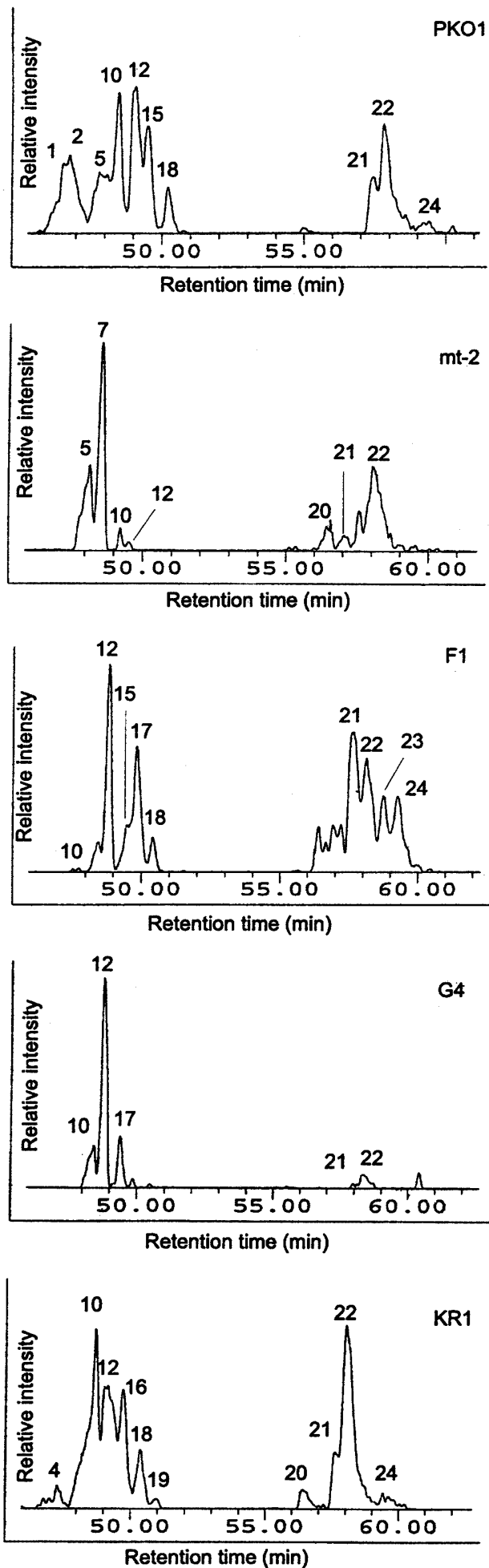

(B)
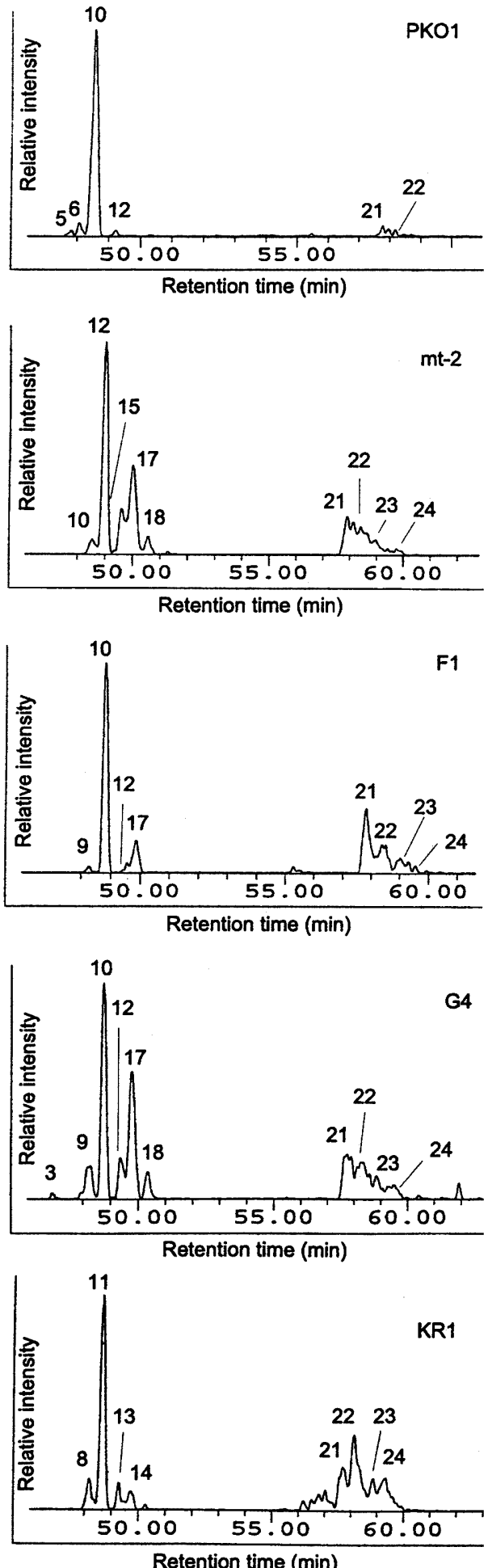
(Fig. 3B), reflecting the overall change in phospholipid composition.

\section{Phospholipid profiles of Pseudomonas putida F1}

PE, PG, and PS were detected in succinate-grown P. putida F1 (Table 1). PE accounted for $54.3 \%$ of the total phospholipids in succinate-grown F1. All of the phospholipids were asymmetrical. 16:0/17:1-PG, which constituted $17.8 \%$ of total phospholipids in F1, was not detected when F1 was grown on toluene. As occurred with $P$. putida $\mathrm{mt}-2$, new phospholipids were synthesized by $\mathrm{F} 1$ when grown on toluene, including a new class of phospholipid (phosphatidylserine). Overall, the composition of phospholipids changed from primary $\mathrm{PE}(\mathrm{PE} /$ $\mathrm{PG}=1.19)$ to $\mathrm{PG}(\mathrm{PE} / \mathrm{PG}=0.67)$. The total concentration of phospholipids decreased substantially (from 194.6 to $26.1 \mathrm{nmol} / \mathrm{ml}$; Fig. 3A), whereas the PRI increased from 17.8 to 19.8 (Fig. 3B).

\section{Phospholipid profiles of Burkholderia cepacia $\mathrm{G} 4$}

The phospholipid composition of succinate-grown G4 was relatively simple, exhibiting five major phospholipids (Table 1). The dominant phospholipid was 16:0/16:1-PG, which constituted $78.9 \%$ of the total phospholipids (Table 1). PE constituted about $6.3 \%$ of the total. The phospholipids composition of G4 significantly changed in response to toluene. For example, PDME containing one or two polyunsaturated fatty acids (C18:2) were found on toluene-grown but not in succinate-grown cells. Exposure to toluene also decreased the relative abundance of 16:0/16:1-PG (from $78.9 \%$ to $30.5 \%$ ) and increased the percentage of $16: 0 /$ $17: 1-\mathrm{PG}$ (from $1.4 \%$ to $22.5 \%$ ). The percentage of PE in total phospholipids also increased from $6.3 \%$ to $29.8 \%$. The total concentration of phospholipids increased (from 56.9 to $88.5 \mathrm{nmol} / \mathrm{ml}$ ), while the phospholipid response index decreased (from 20.5 to 18.2; Fig. 3B) due to toluene exposure.

\section{Phospholipid profiles of Burkholderia pickettii PKO1}

Ten different phospholipids were detected in succinategrown PKO1 (Table 1; Fig. 2). Fatty acids ranged in carbon number from 14 to 18 . The dominant phospholipids were 18:1/16:1-PG (18.7\%), 16:0/16:1-PG $(16.2 \%)$, and $16: 0 / 17: 1-P G(11.8 \%)$. Growth on toluene resulted in dramatic changes in phospholipid composition and concentration, which decreased from 159.8 to $25.4 \mathrm{nmol} / \mathrm{ml}$ (Fig. 3A). Only six phospholipids were detected in toluene-grown cells, among which 16:0/16:1PG was predominant $(86.2 \%)$. As a result, the PE/PG ratio decreased from 0.27 to 0.07 . The PRI increased slightly from 19.9 to 20.7 (Fig. 3B).
Phospholipid profiles of Pseudomonas mendocina KR1

Three phospholipids, 18:1/16:1-PE, 16:0/16:1-PG, and 18:1/16:1-PG, combined constituted $74.1 \%$ of the total lipids of succinate-grown KR1 (Table 1). Phospholipids with polyunsaturated fatty acids 18:2/18:2-PDME were detected in toluene- but not in succinate-grown KR1. This is the only strain besides G4 that contained polyunsaturated fatty acids on both the $s n-1$ and $s n-2$ positions. KR1 also contained exclusively the following phospholipid species when grown on toluene: 18:0/18:0PE, 18:1/18:1-PE, 15:1/16:0-PG, 16:0/16:1-PG, and 17:1/ 18:1-PG. Growth on toluene also decreased the phospholipid concentration (from 214.8 to $77.2 \mathrm{nmol} / \mathrm{ml}$ ) and the PRI (from 19.8 to 16.1) (Fig. 3).

\section{Discussion}

Change in microbial lipid composition in response to membrane-active compounds (e.g., alcohols, phenols, and aromatic compounds) is a well-known phenomenon (Bansal and Khuller 1981; Barrow et al. 1983; Heipieper and de Bont 1994; Heipieper et al. 1992; Ingram 1976, 1977; Pinkart and White 1997; Weber et al. 1994, 1996). Membrane adaptation has been observed to occur in changes of the associated phospholipid fatty acids (not free fatty acids or neutral lipids) (Ingram 1976). Our results indicate that the five pseudomonad strains synthesized different phospholipids when grown on toluene. Specifically, phospholipids with mono- and polyunsaturated fatty acids on one or both of the $s n-1$ and $s n-2$ positions were synthesized.

The total concentration of phospholipids differed substantially in strains grown with and without toluene (Fig. 3A). Toluene-grown strains PKO1, F1, and KR1 exhibited substantially lower phospholipid content, ranging from $13.4 \%$ to $35.9 \%$ of the corresponding content in succinate-grown cells. On the other hand, toluene-grown strains $\mathrm{G} 4$ and mt-2 exhibited relative increases in phospholipid content of $55.5 \%$ and $94.1 \%$, respectively (Fig. 3A). The increase in phospholipid concentration in strains $\mathrm{G} 4$ and $\mathrm{mt}-2$ is consistent with results published by Pinkart and White (1997), who found that the solvent-tolerant strain $P$. putida Idaho increased its total phospholipids by $100 \%$ when exposed to $o$-xylene. Like $P$. putida Idaho, G4 and mt-2 may express a repair mechanism for the replacement of damaged membrane components (Pinkart and White 1997).

The phospholipid response index (PRI) was formulated to reflect how changes in parameters that dictate phospholipid properties (i.e., head group size, chain length, and degree of fatty acid unsaturation) and affect lipid ordering. For example, short chain length, unsaturated fatty acids, and larger head groups are conducive to higher PRI values, which in turn reflect less phospholipid ordering and more fluid membranes. It should be kept in mind, however, that the PRI was defined by 
arbitrarily assigning relative weights to these parameters. Although these weights were assigned based on known biophysical properties, the PRI does not take into account potential synergistic or antagonistic interactions between the parameters considered, or the fact that structural changes may be unevenly distributed. In addition, the cell membrane is a dynamic system where changes in lipid composition may be associated with concurrent changes in proteins that could have a greater effect on membrane properties. Therefore, while the PRI is a convenient index to monitor changes in phospholipid composition and ordering, inferring changes in membrane permeability and function based solely on PRI changes is not advisable.

Both strains that showed increased phospholipid synthesis (i.e., mt-2 and G4) experienced a decrease in PRI values. In contrast, two out of the three strains that showed suppressed phospholipid synthesis (i.e., PKO1 and F1, but not KR1) when grown on toluene experienced an increase in PRI values (Fig. 3B), giving rise to less lipid ordering. The inverse relationship between increased phospholipid synthesis and decreased PRI values suggests that more phospholipids were produced to repair a damaged membrane, and selected phospholipid species that decrease lipid ordering were synthesized to counter the solvent effects of toluene on cell membranes. This is a common bacterial response to such toxic compounds (Bansal and Khuller 1981; Ingram 1977, 1986; Pinkart and White 1997; Taneja and Khuller 1980; Weber et al. 1994, 1996).

Examination of the phospholipid composition of strains G4 and mt-2 indicates that bacteria can exhibit complex response mechanisms in membrane lipids to counter the solvent effect of toluene. For example, strains $\mathrm{mt}-2$ and G4 both exhibited increased phospholipid content when grown on toluene, but experienced opposite changes in head groups. The PE/PG ratio decreased in mt-2 (Table 1 , more PG synthesized), which results in less ordering, while it increased in G4 (i.e., more PE synthesized), which has the opposite effect. Changes in head groups were not the only response mechanism. For example, the synthesis of phospholipids with dipolyunsaturated fatty acids (C18:2) by toluenegrown G4 may play a greater role in maintaining the lipid ordering than synthesis of different classes (head groups) of phospholipid alone. Similar observations were made in previous studies (Ingram 1976; Sullivan et al. 1979) where E. coli exposed to ethanol increased the amount of unsaturated fatty acids as well as the chain length of the fatty acids.

Interestingly, the PG content increased in PKO1 and F1 (Table 1), which is contradictory to previous reports of increased PDME content in $o$-xylene-exposed P. putida MW1200 and P. putida Idaho (Bansal and Khuller 1981; Pinkart and White 1997). It is unknown whether this difference in lipid response reflects dissimilar adaptation mechanisms by different strains, or if different aromatic compounds (toluene vs $o$-xylene) elicit different responses.
In this study, lipid ordering decreased in tolueneexposed PKO1 and F1 (as indicated by the increased PRI values, Fig. 3B), and the increased PG content may have helped to counter the decreased lipid ordering. Overall changes in phospholipid content and composition probably reflect the sensitivity and degree of tolerance of these strains to toxic compounds. The changes in PRI values suggest that different mechanisms were utilized by these strains to maintain optimal lipid ordering in the presence of toluene.

The results presented in Table 1 also have important implications for quantifying microbial concentrations based on phospholipid analyses. The phospholipid esterlinked fatty acids (PLFA) assays have been used to determine microbial biomass in environmental samples (White et al. 1979). The quantification of biomass is based on the premise that phospholipids do not occur in storage lipids, and that their rapid decomposition occurs in dead cells (Balkwill et al. 1988). The total amount of PLFA is converted to total biomass or cell number using conversion factors for surface sediment or subsurface environments (White et al. 1997). Nevertheless, results from this study indicate that the content of fatty acids differs substantially between dissimilar strains and in the same strain grown on different substrates. For example, the phospholipid content of toluene-grown PKO1, F1, and KR1 was $13.4-35.9 \%$ of that found in the same strains grown on succinate, while the phospholipid content of mt-2 and G4 increased by $55.5 \%$ and $94.1 \%$, respectively, when grown on toluene (Table 1). Similar observations were reported in previous studies where PLFA yields varied widely (Haack et al. 1993; Mancuso et al. 1990). Analyses of environmental samples for PLFAs also suggested that toxic contamination in the subsurface induces changes in PLFA content (e.g., higher PLFA content in contaminated areas) (Fang et al. 1997; Smith et al. 1986). Therefore, caution should be exercised in using PLFA or intact phospholipid profiling to determine microbial biomass in environmental samples, particularly when samples from dissimilar environments or locations or distinct organisms are compared.

In summary, all strains exposed to toluene showed significant changes in phospholipid content and composition, as well as considerable diversity in response mechanisms. These changes probably reflect the sensitivity and degree of tolerance of these strains to toluene, and suggest that different mechanisms are utilized by dissimilar bacteria to maintain optimal lipid ordering in the presence of such environmental pollutants.

Acknowledgements The authors wish to thank Randy Simpson (University of Iowa) and Sandy Homola (University of Michigan) for their skillful laboratory assistance. We also thank David T. Gibson for providing $P$. putida $\mathrm{F} 1, P$. putida $\mathrm{mt}-2$, and $P$. mendocina KR1; Jerome J. Kukor for providing $B$. pickettii PKO1; and Malcom S. Shields for providing B. cepacia G4. Partial funding was provided by the Department of Defense's Strategic Environmental Research and Development Program (SERDP) and by the Office of Vice President for Research at the University of Michigan and by a NSF Career Award to Pedro J. Alvarez. 


\section{References}

Balkwill DL, Leach FR, Wilson JT, McNabb JF, White DC (1988) Equivalence of microbial biomass measures based on membrane lipid and cell wall components, adenosine triphosphate and direct counts in subsurface aquifer sediments. Microb Ecol 16: 73-84

Bansal VS, Khuller GK (1981) Changes in phospholipids of $M i$ crosporum species in the presence of ethanol. Arch Microbiol 130: $248-249$

Barrow KD, Collins JG, Rogers PL, Smith GM (1983) Lipid composition of an ethanol-tolerant strain of Zymomonas mobilis. Biochim Biophys Acta 753: 324-330

Beaven MJ, Charpentier C, Rose AH (1982) Production and tolerance of ethanol in relation to phospholipid fatty-acyl composition in Saccharomyces cerevisiae NCYC 431. J Gen Microbiol 128: 1447-1455

Cronan JE, Rock CO (1987) Biosynthesis of membrane lipids. In: Neidhardt FC (ed) Escherichia coli and Salmonella typhimurium, cellular and molecular biology, vol 1. American Society for Microbiology, Washington, DC, pp 474-497

Dombek KM, Ingram LO (1984) Effects of ethanol on the Escherichia coli plasma membrane. J Bacteriol 157: 233-239

Fang J, Barcelona MJ (1998) Structural determination and quantitative analysis of phospholipids using liquid chromatography/ electrospray ionization/mass spectrometry. J Microbiol Methods 33: $23-35$

Fang J, Findlay RH (1996) The use of a classic lipid extraction method for simultaneous recovery of organic pollutants and microbial lipids from sediments. J Microbiol Methods 27: 63-71

Fang J, Barcelona MJ, West C (1997) The use of aromatic acids and phospholipid ester-linked fatty acids for delineation of processes affecting an aquifer contaminated with JP-4 fuel. In: Eganhouse RP (ed) Molecular markers in environmental geochemistry. American Chemical Society, Washington, DC, pp 65-76

Gibson DT, Gschwendt B, Yeh WK, Kobal YM (1973) Initial reactions in the oxidation of ethylbenzene by Pseudomonas putida. Biochemistry 12: 1520-1528

Guckert JB, Hood MA, White DC (1986) Phospholipid esterlinked fatty acid profile changes during nutrient deprivation of Vibrio cholerae: increases in the trans/cis ratio and proportions of cyclopropyl fatty acids. Appl Environ Microbiol 52: 794-801

Haack SK, Garchow H, Odelson DA, Forney LJ, Klug MJ (1993) Accuracy, reproducibility, and interpretation of fatty acid methyl ester profiles of model microbial communities. Appl Environ Microbiol 60: 2483-2493

Heipieper H-J, Bont JAM de (1994) Adaptation of Pseudomonas putida S12 to ethanol and toluene at the level of fatty acid composition of membranes. Appl Environ Microbiol 60: 44404444

Heipieper H-J, Deifenbach R, Keweloh H (1992) Conversion of cis unsaturated fatty acids to trans, a possible mechanism for the protection of phenol-degrading Pseudomonas putida P8 from substrate toxicity. Appl Environ Microbiol 58: 1847-1852

Ingram LO (1976) Adaptation of membrane lipids to alcohols. J Bacteriol 125: 670-678
Ingram LO (1977) Changes in lipid composition of Escherichia coli resulting from growth with organic solvents and food additives. Appl Environ Microbiol 33: 1233-1236

Ingram LO (1986) Microbial tolerance to alcohols: role of cell membrane. Trends Biotechnol 4: 40-44

Mancuso CA, Franzmann PD, Burton HR, Nichols PD (1990) Microbial community structure and biomass estimation of a methanogenic Antarctic lake ecosystem as determined by phospholipid analyses. Microb Ecol 19: 73-95

Melchior DL (1982) Lipid phase transition and regulation of membrane fluidity in prokaryotes. Curr Top Membr Transp 17: 263-316

National Research Council (1993) In situ bioremediation, when does it work? National Academy Press, Washington, DC

Pinkart HC, White DC (1997) Phospholipid biosynthesis and solvent tolerance in Pseudomonas putida strains. Appl Environ Microbiol 179: 4219-4226

Pluschke G, Overath P (1981) Function of phospholipids in Escherichia coli. J Biol Chem 256: 3207-3212

Smith GA, Nickels JS, Kerger BD, Davis JD, Collins SP (1986) Quantitative characterization of microbial biomass and community structure in subsurface material: a prokaryotic consortium responsive to organic contamination. Can J Microbiol 32: 104-111

Stanier RY, Palleroni NJ, Doudoroff M (1966) The aerobic pseudomonads: a taxonomic study. J Gen Microbiol 43: 159-271

Stubbs CD, Kouyama T, Kinosita K, Ikegami A (1981) Effect of double bonds on the dynamic properties of hydrocarbon region of lecithin bilayers. Biochemistry 20: 4257-4262

Sullivan KH, Hegeman GD, Cordes EH (1979) Alteration of the fatty acid composition of Escherichia coli by growth in the presence of normal alcohols. J Bacteriol 138: 133-138

Taneja R, Khuller GK (1980) Ethanol-induced alterations in phospholipids and fatty acids of Mycobacterium smegmatis ATCC 607. FEMS Microbiol Lett 8: 83-85

Tursman JF, Cork DJ (1992) Subsurface contaminant bioremediation engineering. Crit Rev Environ Control 22: 1-26

Weber FJ, Isken S, Bont JAM de (1994) Cis/trans isomerization of fatty acids as a defense mechanisms of Pseudomonas putida strains to toxic concentrations of toluene. Microbiology 140: 2013-2017

Weber FJ, Isken S, Bont JAM de (1996) Adaptation mechanisms of microorganisms to the toxic effects of organic solvents on membranes. Biochim Biophysica Acta 1286: 225-245

White DC, Bobbie RJ, King JD, Nickels JS, Amoe P (1979) Lipid analysis of sediments for microbial biomass and community structure. In: Litchfield CD, Seyfried PL (eds) Methodology for biomass determination and microbial activities in sediments, ASTM STP 673. American Society for Testing and Materials, Philadelphia, Pa, pp 87-103

White DC, Pinkart, HC, Ringelberg AB (1997) Biomass measurements: biochemical approaches. In: Hurst CJ, Knudson GR, McInerney MJ, Stetzenbach LD, Walter MV (eds) Manual of environmental microbiology. ASM Press, Washington, DC, pp 91-101 